\title{
InfluênCia do Glyphosate sobre a Nodulação e o Crescimento de Cultivares de Soja Resistente ao Glyphosate ${ }^{1}$
}

\author{
Glyphosate Influence on Growth and Nodulation of Glyphosate Resistant Soybean Cultivars
}

\author{
OLIVEIRA JR., R.S..$^{*}$, DVORANEN, E.C..$^{*}$, CONSTANTIN, J..$^{2^{*}}$, CAVALIERI, S.D..$^{{ }^{*}}$, \\ FRANCHINI, L.H.M. ${ }^{*}$, RIOS, F.A. ${ }^{4^{*}}$ e BLAINSKI, E. ${ }^{4^{*}}$
}

\begin{abstract}
RESUMO - A soja resistente ao glyphosate (RR) é uma tecnologia que vem acrescentar mais uma ferramenta ao manejo de plantas daninhas para essa cultura, que possui a maior área plantada em nosso país. Por se tratar de uma técnica recente tanto no Brasil quanto no mundo, é preciso estudos buscando informações para o uso correto desta importante e cada vez mais freqüente prática agrícola: o cultivo de soja transgênica. Objetivou-se com este trabalho avaliar os efeitos causados pelas aplicações de glyphosate sobre 20 cultivares de soja RR. As doses utilizadas foram: testemunha sem herbicida; glyphosate em aplicação seqüencial de $0,54 / 0,36 \mathrm{~kg}$ equivalente ácido (e.a.) ha ${ }^{-1}$, aos 12 / 24 dias após a emergência (DAE); glyphosate em aplicação única de 0,72 kg e.a. ha ${ }^{-1}$, aos 20 DAE; glyphosate em aplicação seqüencial de 0,72/0,54 $\mathrm{kg} \mathrm{ha}^{-1}$, aos 12/24 DAE; e glyphosate em aplicação única de $0,90 \mathrm{~kg} \mathrm{ha}^{-1}$, aos $24 \mathrm{DAE}$. Foram avaliadas as variáveis matéria seca do sistema radicular (MSSR), matéria seca da parte aérea (MSPA), matéria seca dos nódulos acumulados (MSNT) e número de nódulos acumulados (NN). Os cultivares que demonstraram maior suscetibilidade às aplicações de glyphosate foram: MSOY 8008 RR, ANTA RR, CD 213 RR, MSOY 8100 RR, VALIOSA RR, CD 219 RR, MSOY 6001 RR, CRISTALINA RR e BRS 247 RR, apresentando reduções de pelo menos três das quatro variáveis estudadas. BRS 242 RR, BRS 243 RR, BRS 244 RR, BRS 246 RR, CD 214 RR, MSOY 8151 RR, MSOY 9000 RR e BRS 245 RR foram as mais tolerantes, pois não sofreram reduções significativas em nenhuma ou em uma das variáveis avaliadas. De modo geral, a MSSR foi mais afetada pela modalidade de aplicação do que pela dose de glyphosate; por outro lado, a MSPA e a nodulação foram mais afetadas em cultivares de ciclo mais longo, em relação às variedades de ciclo precoce. A nodulação também foi menos afetada pelo glyphosate em variedades do grupo BRS, comparada aos demais materiais genéticos.
\end{abstract}

Palavras-chave: fixação biológica do $\mathrm{N}_{2}$, herbicida, rhizobium, simbiose

\begin{abstract}
Glyphosate resistant soybean (GR) is a new technological tool in weed management applied in soybean culture, which accounts for the largest area planted in Brazil. Since it has been only recently applied in Brazil and abroad, further studies are needed to search for useful information on the correct use of this important and increasingly applied agricultural practice: the cultivation of GR soybean. Thus, the aim of this work was to evaluate the effects caused by glyphosate applications on twenty GR soybean cultivars. The rates applied were: check without herbicide; sequential application of glyphosate at $0.54 / 0.36 \mathrm{~kg}$ acid equivalent (AE) ha ${ }^{1}$, at 12/24 days after emergence (DAE); single application of glyphosate at $0.72 \mathrm{~kg}$ a.i. hat , at $20 \mathrm{DAE}$; sequential application of glyphosate at $0.72 / 0.54 \mathrm{~kg}$ a.i. ha $\mathrm{h}^{-1}$, at 12/24 DAE; single application of glyphosate at $0.90 \mathrm{~kg}$
\end{abstract}

1 Recebido para publicação em 9.11.2007 e na forma revisada em 6.6.2008.

Parte da dissertação do segundo autor apresentada para obtenção do título de Mestre em Agronomia, Área de Concentração em Proteção de Plantas - Programa de Pós-Graduação em Agronomia - Universidade Estadual de Maringá (UEM).

2 Professor Associado do Dep. de Agronomia - Núcleo de Estudos Avançados em Ciência das Plantas Daninhas (NAPD/UEM) Av. Colombo, 5790, 87020-900, Maringá-PR; ${ }^{3}$ Engo-Agroo - , M.Sc., Universidade Estadual de Maringá - UEM; ${ }^{4}$ Graduando em Agronomia - UEM, Bolsista de Iniciação Científica do NAPD/UEM; * Bolsista do CNPq.

Planta Daninha, Viçosa-MG, v. 26, n. 4, p. 831-843, 2008 
ha ${ }^{-1}$, at $24 D A E$. The variables root dry weight (RDW), shoot dry weight (SDW), accumulated nodule dry weight (ANDW) and number of accumulated nodules (NAN) were evaluated. The cultivars MSOY 8008 RR, ANTA RR, CD 213 RR, MSOY 8100 RR, VALIOSA RR, CD 219 RR, MSOY 6001 $R R, C R I S T A L I N A R R$ and BRS $247 R R$ showed the highest susceptibility to the glyphosate applications, with reductions in at least three out of the four variables studied. BRS $242 R R, B R S$ 243 RR, BRS 244 RR, BRS 246 RR, CD 214 RR, MSOY 8151 RR, MSOY 9000 RR and BRS 245 RR showed the highest tolerance, with no significant reductions or only in one of the four assessed variables. In general, RDWwas more affected by the mode of application than the glyphosate rate; however, SDW and nodulation (ANDW and NAN) were more affected in the longer cycle cultivars, compared to the earlier cycle varieties. Nodulation was also less affected by glyphosate in the $B R S$ variety group, compared to other genetic materials.

Keywords: $\quad \mathrm{N}_{2}$ biological fixation, herbicide, rhizobium, symbiosis.

\section{INTRODUÇÃO}

O glyphosate é um herbicida não-seletivo, de amplo espectro, aplicado em pós-emergência, que controla a maioria das espécies daninhas. Esse herbicida inibe a sintese de aminoácidos aromáticos (fenilalanina, tirosina e triptofano) em plantas e microrganismos que possuem o ciclo do ácido shiquímico (Jaworski, 1972; Fisher et al., 1986). O mecanismo de ação do glyphosate é a inibição da enzima EPSPS, que catalisa a condensação do ácido shiquímico a fosfoenolpiruvato (Oliveira Jr., 2001).

No entanto, nem todos os organismos respondem da mesma forma a este e a outros herbicidas, como foi constatado por Kapusta \& Rouwenhorst (1973), Alagavadi \& Reddy (1986), Kishinevsky et al. (1988) e Delannay et al. (1995). Santos et al. (2004) estudaram os efeitos de formulações comerciais de glyphosate sobre estirpes de Bradyrhizobium japonicum (SEMIA 5079) e Bradyrhizobium elkanii (SEMIA 5019 e SEMIA 587), em condições de laboratório com dose de $0,26 \mathrm{mM}$ do equivalente ácido (EA), e constataram diferenças entre as estirpes de rizóbios, assim como entre as formulações de glyphosate. A estirpe menos tolerante à maioria das formulações testadas foi a SEMIA 587, e a mais sensivel ao glyphosate padrão, sem adição de sais ou de outros aditivos, foi a SEMIA 5019. Estudos desenvolvidos por Moorman et al. (1992) mostraram inibição diferencial de crescimento entre estirpes de $B$. japonicum expostas a $0,5 \mathrm{mM}$ de glyphosate e inibição de todas as estirpes testadas quando a dose foi elevada para $5 \mathrm{mM}$.
O efeito tóxico do glyphosate sobre os nódulos pode ser atribuído a: (1) inabilidade das plantas em sintetizar aminoácidos aromáticos, visto que a EPSP sintase está inibida; 2) um dreno de energia resultante de ATP e fosfoenolpiruvato gastos no acúmulo de ácido shiquímico, ácido heptulossômico-7-fosfato-3desoxiarabinose e ácidos hidroxibenzóicos; e 3) toxicidade dos intermediários da via do ácido shiquímico, acumulados pela inibição da EPSP sintase (Bellaloui et al., 2006).

Nos Estados Unidos, centenas de variedades de soja transgênica resistente ao glyphosate (RR), de diferentes grupos de maturação, encontram-se disponiveis no mercado. Segundo Zablotowicz \& Reddy (2004), as respostas fisiológicas dessas variedades às aplicações de glyphosate podem variar em função de fatores como localização geográfica, condições ambientais, tipos de solo, população de rizóbios, entre outros.

No Brasil, o plantio da soja resistente ao glyphosate foi legalmente autorizado em setembro de 2003, pela Medida Provisória no 131 , que no mesmo ano foi convertida na Lei no 10.184 , de 15 de dezembro de 2003. No entanto, a liberação para cultivo e comercialização da soja resistente a glyphosate gerou e ainda gera polêmica. Apesar dos impasses legais, segundo o relatório anual de 2006 do Serviço Internacional para a Aquisição de Aplicações em Agrobiotecnologia (ISAAA), no Brasil a área plantada com soja transgênica na safra 2005/06 foi de 11,4 milhões de hectares, ocupando a terceira posição entre os países que mais cultivam transgênicos no mundo, ficando 
atrás apenas dos Estados Unidos e da Argentina. Atualmente, o Brasil conta com aproximadamente 90 variedades de soja $R R$, de várias empresas de sementes, disponiveis para plantio. Entretanto, poucos estudos sobre os efeitos do glyphosate nas variedades RR cultivadas no Brasil foram desenvolvidos, assim como sobre sua influência na população de microrganismos do solo. Há, dessa forma, uma lacuna de conhecimento a respeito do comportamento dessas variedades em relação ao glyphosate.

Desse modo, este estudo visou avaliar, em condições de casa de vegetação, a influência do glyphosate sobre a nodulação e o desenvolvimento da parte aérea e sistema radicular em 20 variedades de soja transgênica $R R$ cultivadas no País.

\section{MATERIAL E MÉTODOS}

O experimento foi conduzido em casa de vegetação do Laboratório de Plantas Daninhas da Universidade Estadual de Maringá, no município de Maringá, que se situa na região noroeste do Estado do Paraná, a 2325'31" de latitude sul, 51 ${ }^{\circ} 56^{\prime} 19^{\prime \prime}$ de longitude oeste de Greenwich e a $542 \mathrm{~m}$ de altitude. O período de tempo compreendido entre o início e o fim do experimento foi de 19/12/2005 a 25/3/2006.

No trabalho foram avaliadas 19 variedades de soja transgênica resistente ao glyphosate, provenientes das regiões Sul, Sudeste e Centro-Oeste do Brasil, e uma proveniente da
Argentina. As variedades utilizadas foram BRS 245 RR; MSOY 8151 RR; AL 83 TROPICAL RR; CD 214 RR; BRS 242 RR; BRS 244 RR; CD 219 RR; MSOY 8008 RR; ANTA RR; BRS 243 RR; MSOY 6001 RR; CRISTALINA RR; BRS 247 RR; MSOY 9000 RR; CD 213 RR; MSOY 8100 RR; VALIOSA RR; MSOY 8000 RR; BRS 246 RR; e Não-Identificada (NI). Os cultivares, os grupos de maturação e as respectivas procedências encontram-se na Tabela 1 .

As unidades experimentais foram vasos de polietileno de $5 \mathrm{dm}^{3}$, onde foram semeadas dez sementes de cada variedade a $3 \mathrm{~cm}$ de profundidade. Os vasos foram preenchidos com solo de textura franco-argilo-arenosa no horizonte A de onde foi coletado, após peneiramento (10 mesh). Os resultados das análises química e granulométrica encontram-se na Tabela 2. A semeadura foi efetuada com sementes tratadas com [Carbendazim+Thiram] + Imidacloprid nas doses de [30+70] g i.a. + 60 g para cada $100 \mathrm{~kg}$ de sementes e posteriormente inoculadas com inoculante da marca comercial Rhizofix, formulação turfosa, produzido com as estirpes de Bradyrhizobium elkanii, SEMIA 587 e SEMIA 5019, na concentração de 3 × $10^{9}$ rizóbios por grama, na dose de 400 g p.c. por $100 \mathrm{~kg}$ de sementes.

Após a semeadura, a superfície do solo foi coberta com palha de aveia picada, com o equivalente a $5.000 \mathrm{~kg} \mathrm{ha}^{-1}$. Quando as plantas atingiram o estádio $\mathrm{V} 1$, foi feito um raleamento, mantendo-se duas plantas por vaso.

Tabela 1 - Variedades de soja RR, grupos de maturação e procedência

\begin{tabular}{|c|c|c|c|c|c|c|c|c|c|c|}
\hline Variedade & $\begin{array}{c}\text { BRS } 245 \\
\text { RR }\end{array}$ & $\begin{array}{c}\text { MSOY } \\
8151 \text { RR }\end{array}$ & $\begin{array}{c}\text { AL } 83 \\
\text { TROPICAL } \\
\text { RR }\end{array}$ & $\begin{array}{c}\text { CD } 214 \\
\text { RR }\end{array}$ & $\begin{array}{c}\text { BRS } 242 \\
\text { RR }\end{array}$ & $\begin{array}{c}\text { BRS } 244 \\
\text { RR }\end{array}$ & $\begin{array}{c}\text { CD } 219 \\
\text { RR }\end{array}$ & $\begin{array}{c}\text { MSOY } \\
8008 \text { RR }\end{array}$ & $\begin{array}{c}\text { ANTA } \\
\text { RR }\end{array}$ & $\begin{array}{c}\text { BRS } \\
243 \mathrm{RR}\end{array}$ \\
\hline $\begin{array}{l}\text { Grupo de } \\
\text { maturação }\end{array}$ & $\begin{array}{l}\text { Semi- } \\
\text { precoce }\end{array}$ & $\begin{array}{l}\text { Semi- } \\
\text { tardio }\end{array}$ & $\begin{array}{l}\text { Semi- } \\
\text { tardio }\end{array}$ & Precoce & Precoce & $\begin{array}{l}\text { Semi- } \\
\text { precoce }\end{array}$ & Médio & $\begin{array}{l}\text { Semi- } \\
\text { tardio }\end{array}$ & - & Precoce \\
\hline Procedência & $\begin{array}{c}\text { Toledo } \\
\text { PR }\end{array}$ & $\begin{array}{c}\text { Goiânia } \\
\text { GO }\end{array}$ & $\begin{array}{l}\text { Rondonó- } \\
\text { polis } \\
\text { MT }\end{array}$ & $\begin{array}{c}\text { Toledo } \\
\text { PR }\end{array}$ & $\begin{array}{c}\text { Toledo } \\
\text { PR }\end{array}$ & $\begin{array}{c}\text { Toledo } \\
\text { PR }\end{array}$ & $\begin{array}{c}\text { Jataí } \\
\text { GO }\end{array}$ & $\begin{array}{c}\text { Goiânia } \\
\text { GO }\end{array}$ & $\begin{array}{c}\text { Ponta } \\
\text { Grossa } \\
\text { PR }\end{array}$ & $\begin{array}{l}\text { Toledo } \\
\text { PR }\end{array}$ \\
\hline Variedade & $\begin{array}{c}\text { MSOY } \\
6001 \mathrm{RR}\end{array}$ & $\begin{array}{c}\text { CRISTA } \\
\text { LINA RR }\end{array}$ & $\begin{array}{c}\text { BRS } 247 \\
\text { RR }\end{array}$ & $\begin{array}{c}\text { MSOY } \\
9000 \mathrm{RR}\end{array}$ & $\begin{array}{c}\text { CD } 213 \\
\text { RR }\end{array}$ & $\begin{array}{c}\text { MSOY } \\
8100 \text { RR }\end{array}$ & $\begin{array}{c}\text { Valiosa } \\
\text { RR }\end{array}$ & $\begin{array}{c}\text { MSOY } \\
8000 \text { RR }\end{array}$ & $\begin{array}{c}\text { BRS } 246 \\
\text { RR }\end{array}$ & NI* \\
\hline $\begin{array}{l}\text { Grupo de } \\
\text { maturação }\end{array}$ & Precoce & Tardio & Médio & Tardio & Precoce & $\begin{array}{l}\text { Semi- } \\
\text { tardio }\end{array}$ & Tardio & $\begin{array}{l}\text { Semi- } \\
\text { tardio }\end{array}$ & $\begin{array}{l}\text { Semi- } \\
\text { precoce }\end{array}$ & - \\
\hline Procedência & $\begin{array}{c}\text { Toledo } \\
\text { PR }\end{array}$ & $\begin{array}{c}\text { Chapadão } \\
\text { do Sul } \\
\text { MS }\end{array}$ & $\begin{array}{c}\text { Toledo } \\
\text { PR }\end{array}$ & $\begin{array}{c}\text { Chapadão } \\
\text { do Sul } \\
\text { MS }\end{array}$ & $\begin{array}{c}\text { Ponta } \\
\text { Grossa } \\
\text { PR }\end{array}$ & $\begin{array}{c}\text { Ponta } \\
\text { Grossa } \\
\text { PR }\end{array}$ & $\begin{array}{l}\text { Adaman- } \\
\text { tina SP }\end{array}$ & $\begin{array}{c}\text { Goiânia } \\
\text { GO }\end{array}$ & $\begin{array}{c}\text { Toledo } \\
\text { PR }\end{array}$ & $\begin{array}{c}\text { Argen- } \\
\text { tina }\end{array}$ \\
\hline
\end{tabular}

* NI = não-identificada.

- Dados não disponíveis. 
Tabela 2 - Resultado das análises* química e granulométrica do solo utilizado no experimento (camada de 0-20 cm de profundidade). Maringá-PR, 2006

\begin{tabular}{|c|c|c|c|c|c|c|c|c|c|c|}
\hline \multicolumn{2}{|c|}{$\mathrm{pH}$} & $\mathrm{Al}^{3+}$ & $\mathrm{H}^{+}+\mathrm{Al}^{3+}$ & $\mathrm{Ca}^{2+}$ & $\mathrm{Mg}^{2+}$ & $\mathrm{K}^{+}$ & SB & CTC & \multirow{2}{*}{$\frac{\mathrm{P}}{\mathrm{mg} \mathrm{dm^{-3 }}}$} & \multirow{2}{*}{$\frac{\mathrm{C}}{\mathrm{g} \mathrm{dm}^{-3}}$} \\
\hline $\mathrm{CaCl}_{2}$ & $\mathrm{H}_{2} \mathrm{O}$ & \multicolumn{7}{|c|}{ - } & & \\
\hline 5,2 & 5,8 & 0,0 & 3,68 & 3,91 & 1,95 & 0,40 & 6,26 & 9,94 & 3,0 & 6,97 \\
\hline \multicolumn{3}{|c|}{ Areia grossa } & \multicolumn{3}{|c|}{ Areia fina } & \multicolumn{3}{|c|}{ Silte } & \multicolumn{2}{|c|}{ Argila } \\
\hline \multicolumn{11}{|c|}{ 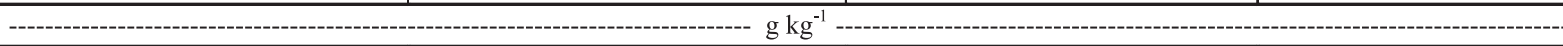 } \\
\hline \multicolumn{3}{|c|}{370} & \multicolumn{3}{|c|}{320} & \multicolumn{3}{|c|}{80} & \multicolumn{2}{|c|}{230} \\
\hline
\end{tabular}

* Realizadas no Laboratório de Solos - UEM - Maringá

O delineamento utilizado foi em blocos ao acaso, com sete repetições. Os tratamentos resultaram do arranjo fatorial entre as vinte variedades de soja $R R$ e cinco modalidades de aplicação de glyphosate. Os niveis das aplicações com glyphosate foram: zero (testemunha sem herbicida); aplicação seqüencial de glyphosate: 0,54/0,36 kg e.a. ha- ${ }^{-1}(12$ e 24 DAE) (seqüencial I); aplicação única de glyphosate: 0,72 kg e.a. ha ${ }^{-1}$ (20 DAE) (dose única I); aplicação seqüencial de glyphosate: 0,72/0,54 kg e.a. ha-1 (12 e $24 \mathrm{DAE}$ ) (seqüencial II); e aplicação única de glyphosate: $0,90 \mathrm{~kg}$ e.a. ha-1 (24 DAE) (dose única II). As doses das aplicações com glyphosate estão em $\mathrm{kg}$ de equivalente ácido de N-(fosfonometil) glicina - glyphosate por hectare. A lista com as modalidades de aplicação, doses, épocas de aplicação de glyphosate e estádios de desenvolvimento da soja é apresentada na Tabela 3.

As aplicações foram feitas com os vasos colocados do lado de fora da casa de vegetação, utilizando-se barra equipada com quatro bicos com pontas tipo leque (Teejet XR 110.02), espaçados a $50 \mathrm{~cm}$ entre si, posicionada na altura de $50 \mathrm{~cm}$ da superficie dos vasos e volume relativo de calda de $200 \mathrm{~L} \mathrm{ha}^{-1}$. Por ocasião das aplicações, as condições de temperatura estavam entre 23 e $29^{\circ} \mathrm{C}$, umidade relativa do ar entre 74 e $89 \%$, solo úmido, velocidade do vento entre 5 e $10 \mathrm{~km} \mathrm{~h}^{-1}$ e céu aberto sem nebulosidade. Após as aplicações do herbicida, os vasos foram levados de volta à casa de vegetação e irrigados apenas no dia seguinte, garantindo assim a absorção foliar do herbicida. Os vasos foram irrigados diariamente, de forma a manter o solo úmido, e mantidos livres de plantas daninhas por meio de capinas manuais.

Ao atingirem o estádio R3, as plantas foram cortadas rente ao solo, embaladas em sacos de papel e secas em estufa de circulação forçada de ar a $65-70{ }^{\circ} \mathrm{C}$ até peso constante, quando foram tomados os pesos da matéria seca, por pesagem em balança de precisão. As raízes foram lavadas em água corrente e, em seguida, foi feito o destaque e a contagem do número de nódulos. Da mesma forma, foi feita a secagem das raízes e dos nódulos para posterior determinação da sua matéria seca. Foi avaliada a matéria seca de parte aérea, do sistema radicular, do total de nódulos acumulados e o número de nódulos por vaso.

Os erros dos dados passaram pelos testes de Levene e Shapiro-Wilk, com o objetivo de avaliar a sua variância e normalidade. Os dados foram transformados em porcentagem em relação à testemunha, a fim de que se obtivesse homocedasticidade e normalidade dos erros. Todos os dados foram submetidos à análise de variância e ao teste de agrupamento de médias Scott-Knott a $5 \%$ de

Tabela 3 - Modalidades de aplicação com glyphosate, doses (kg e.a. ha-1), épocas de aplicação e estádios de desenvolvimento da soja

\begin{tabular}{|l|c|c|c|}
\hline \multicolumn{1}{|c|}{ Modalidade de aplicação } & Dose em kg e.a. ha ${ }^{-1}$ & Época de aplicação & Estádio da soja \\
\hline Testemunha sem aplicação & - & - & - \\
\hline Aplicação seqüencial I (SI) & $0,54 / 0,36$ & $12 / 24$ DAE & V3/V5 \\
\hline Aplicação em dose única I (DUI) & 0,72 & 20 DAE & V4 \\
\hline Aplicação seqüencial II (SII) & $0,72 / 0,54$ & $12 / 24$ DAE & V3/V5 \\
\hline Aplicação em dose única II (DUII) & 0,90 & 24 DAE & V5 \\
\hline
\end{tabular}


probabilidade, pelo SISVAR (Ferreira, 2000). Posteriormente, os cultivares foram agrupados por materiais genéticos, grupos de maturação e modalidades de aplicação e submetidos a testes por contrastes. Os contrastes analisados foram:

$$
\begin{aligned}
& \hat{C}_{1}=\hat{\mu}_{B R S 242}+\hat{\mu}_{B R S 243}+\hat{\mu}_{B R S 244}+\hat{\mu}_{B R S 245} \\
& +\hat{\mu}_{B R S 246}+\hat{\mu}_{B R S 247}-\hat{\mu}_{M S O Y 6001}-\hat{\mu}_{M S O Y} 8000 \\
& -\hat{\mu}_{M S O Y ~ 8008}-\hat{\mu}_{M S O Y ~ 8100}-\hat{\mu}_{M S O Y ~ 8151} \\
& -\hat{\mu}_{M S O Y 9000} \text {; } \\
& \hat{C}_{2}=\hat{\mu}_{B R S 242}+\hat{\mu}_{B R S 243}+\hat{\mu}_{B R S 244}+\hat{\mu}_{B R S 245} \\
& +\hat{\mu}_{B R S 246}+\hat{\mu}_{B R S 247}-2 \hat{\mu}_{C D 213}-2 \hat{\mu}_{C D 214} \\
& -2 \hat{\mu}_{C D 219} \text {; } \\
& \hat{C}_{3}=\hat{\mu}_{M S O Y ~ 6001}+\hat{\mu}_{M S O Y 8000}+\hat{\mu}_{M S O Y 8008} \\
& +\hat{\mu}_{M S O Y 8100}+\hat{\mu}_{M S O Y 8151}+\hat{\mu}_{M S O Y 9000} \\
& -2 \hat{\mu}_{C D 213}-2 \hat{\mu}_{C D 214}-2 \hat{\mu}_{C D 219} ; \\
& \hat{C}_{4}=\hat{\mu}_{B R S 242}+\hat{\mu}_{B R S 243}+\hat{\mu}_{B R S 244}+\hat{\mu}_{B R S 245} \\
& +\hat{\mu}_{B R S 246}+\hat{\mu}_{C D 213}+\hat{\mu}_{C D 214}+\hat{\mu}_{M S O Y 6001} \\
& -\hat{\mu}_{B R S 247}-\hat{\mu}_{C D 219}-\hat{\mu}_{M S O Y 8000}-\hat{\mu}_{M S O Y 8008} \\
& -\hat{\mu}_{M S O Y} 8100-\hat{\mu}_{M S O Y} 8151-\hat{\mu}_{\text {MSOY 9000 }}-\hat{\mu}_{\text {VALIOSA }} \text {; } \\
& \hat{C}_{5}=\hat{\mu}_{S I}-\hat{\mu}_{S I I} \text {; } \\
& \hat{C}_{6}=\hat{\mu}_{D U I}-\hat{\mu}_{D U I I} \text {; } \\
& \hat{C}_{7}=\hat{\mu}_{S I}+\hat{\mu}_{S I I}-\hat{\mu}_{D U I}-\hat{\mu}_{D U I I} \text {; } \\
& \hat{C}_{8}=\hat{\mu}_{S I}+\hat{\mu}_{D U I}-\hat{\mu}_{S I I}-\hat{\mu}_{D U I I} \text {; } \\
& \hat{C}_{9}=2 \hat{\mu}_{\text {TESTEMUNHA }}-\hat{\mu}_{S I}-\hat{\mu}_{S I I} \text {; } \\
& \hat{C}_{10}=2 \hat{\mu}_{\text {TESTEMUNHA }}-\hat{\mu}_{D U I}-\hat{\mu}_{\text {DUII }} \text {; }
\end{aligned}
$$

de modo que $\hat{C}$ é a estimativa do contraste e $\hat{\mu}$, a média de cada tratamento.

Os cultivares, as modalidades de aplicação e os respectivos contrastes formados estão apresentados na Tabela 4. O objetivo da comparação dos dados por contraste foi criar grupos com semelhanças entre si, para facilitar a análise dos efeitos dos cultivares e dos tratamentos sobre as variáveis-respostas avaliadas.

\section{RESULTADOS E DISCUSSÃO}

Observou-se efeito de redução de matéria seca de nódulos totais (MSNT) acumulados (Tabela 5) em plantas das variedades CD 219 RR, MSOY 8008 RR, ANTA RR, MSOY 6001 RR, CRISTALINA RR, CD 213 RR, MSOY 8100 RR, VALIOSA RR, MSOY 8000 RR e NI. A variedade CRISTALINA RR teve a MSNT afetada pelas duas modalidades de aplicação seqüencial (SI e SII) e pela dose única mais alta (DUII). A variedade CD $213 \mathrm{RR}$ foi a que sofreu maior influência das aplicações de glyphosate em relação à testemunha. Para esta variedade, os tratamentos com dose maior (SII e DUII) reduziram em até $61,4 \%$ a MSNT, e os tratamentos com dose menor (SI e DUI), em até $35,8 \%$. As variedades CD 219 RR, MSOY 8008 RR, ANTA RR, MSOY 6001 RR, MSOY 8100 RR, VALIOSA RR, MSOY 8000 RR e NI tiveram a MSNT reduzida em intensidade semelhante, em relação à testemunha, por todas as modalidades de aplicação de glyphosate (SI, DUI, SII e DUII), porém as aplicações herbicidas não diferiram entre si. As variedades BRS 245 RR, MSOY 8151 RR, AL 83 TROPICAL RR, CD 214 RR, BRS 242 RR, BRS 244 RR, BRS 243 RR, BRS 247 RR, MSOY 9000 RR e BRS 246 RR não tiveram as respectivas MSNT afetadas por nenhuma das modalidades de aplicação, quando comparadas com a testemunha.

A comparação entre os cultivares mostrou diferença apenas na aplicação da maior dose única. Os cultivares MSOY 8008 RR, MSOY 6001 RR e CD 213 RR apresentaram maior redução em relação aos outros.

Jaworski (1972), Eberbach \& Douglas (1989), Liu et al. (1991), Martensson (1992), Moorman et al. (1992), Hernandez et al. (1999) e Reddy et al. (2000) encontraram efeitos de inibição de crescimento de rizóbios e de nodulação em plantas de diversas espécies tratadas com glyphosate. Esses efeitos variaram de acordo com doses, espécies e épocas de aplicação. Os resultados encontrados no presente trabalho se assemelham a esses e sugerem que a resposta às aplicações não é uniforme em relação aos materiais testados nem no tocante com a modalidade de aplicação. 
Tabela 4 - Contrastes entre cultivares e modalidades de aplicação

\begin{tabular}{|c|c|c|c|c|c|c|}
\hline \multirow{2}{*}{\multicolumn{4}{|c|}{ Contraste }} & \multicolumn{3}{|c|}{ Componente de cada grupo no contraste } \\
\hline & & & & \multicolumn{3}{|c|}{ Cultivares } \\
\hline$\hat{C}_{1}$ & $\begin{array}{l}\text { Cultivares } \\
\text { BRS }\end{array}$ & vs & $\begin{array}{l}\text { Cultivares } \\
\text { Monsoy }\end{array}$ & $\begin{array}{c}\text { BRS } 242 \text { RR, BRS } 243 \text { RR, BRS } 244 \\
\text { RR, BRS } 245 \text { RR, BRS } 246 \text { RR e } \\
\text { BRS 247RR }\end{array}$ & $v s$ & $\begin{array}{l}\text { MSOY } 6001 \text { RR, MSOY } 8000 \mathrm{RR} \text {, } \\
\text { MSOY } 8008 \text { RR, MSOY } 8100 \mathrm{RR} \text {, } \\
\text { MSOY } 8151 \text { RR e MSOY } 9000 \mathrm{RR}\end{array}$ \\
\hline$\hat{C}_{2}$ & $\begin{array}{l}\text { Cultivares } \\
\text { BRS }\end{array}$ & vs & Cultivares CD & $\begin{array}{c}\text { BRS } 242 \text { RR, BRS } 243 \text { RR, BRS } 244 \\
\text { RR, BRS } 245 \text { RR, BRS } 246 \text { RR e } \\
\text { BRS 247RR }\end{array}$ & $v s$ & $\begin{array}{c}\text { CD } 213 \text { RR, CD } 214 \text { RR e CD } 219 \\
\text { RR }\end{array}$ \\
\hline$\hat{C}_{3}$ & $\begin{array}{l}\text { Cultivares } \\
\text { Monsoy }\end{array}$ & $v s$ & Cultivares CD & $\begin{array}{l}\text { MSOY } 6001 \mathrm{RR}, \text { MSOY } 8000 \mathrm{RR}, \\
\text { MSOY } 8008 \text { RR, MSOY } 8100 \mathrm{RR}, \\
\text { MSOY } 8151 \text { RR e MSOY } 9000 \mathrm{RR}\end{array}$ & $v s$ & $\begin{array}{c}\text { CD } 213 \text { RR, CD } 214 \text { RR e CD } 219 \\
\text { RR }\end{array}$ \\
\hline$\hat{C}_{4}$ & $\begin{array}{l}\text { Cultivares } \\
\text { precoces }\end{array}$ & vs & $\begin{array}{l}\text { Cultivares não } \\
\text { precoces }\end{array}$ & $\begin{array}{c}\text { BRS } 242 \text { RR, BRS } 243 \text { RR, BRS } 244 \\
\text { RR, BRS 245 RR, BRS } 246 \text { RR, CD } \\
213 \text { RR, CD } 214 \text { RR e MSOY } 6001 \\
\text { RR }\end{array}$ & vs & $\begin{array}{c}\text { BRS } 247 \text { RR, CD } 219 \text { RR, MSOY } \\
8000 \text { RR, MSOY } 8008 \text { RR, MSOY } \\
\text { 8100 RR, MSOY } 8151 \text { RR e } \\
\text { MSOY } 9000 \text { RR e VALIOSA RR }\end{array}$ \\
\hline & & & & \multicolumn{3}{|c|}{ Modalidade de aplicação } \\
\hline$\hat{C}_{5}$ & SI & vs & SII & $0,54 / 0,36 \mathrm{~kg}$ e.a. $\mathrm{ha}^{-1}(12$ e $24 \mathrm{DAE})$ & $v s$ & $\begin{array}{c}\text { 0,72/0,54 kg e.a. ha- }{ }^{-1}(12 \text { e } 24 \\
\text { DAE) }\end{array}$ \\
\hline$\hat{C}_{6}$ & DUI & $v s$ & DUII & $0,72 \mathrm{~kg}$ e.a. ha $^{-1}(20 \mathrm{DAE})$ & $v s$ & $0,90 \mathrm{~kg}$ e.a. $\mathrm{ha}^{-1}(24 \mathrm{DAE})$ \\
\hline$\hat{C}_{7}$ & Seqüenciais & $v s$ & Doses Únicas & SI e SII & $v s$ & DUI e DUII \\
\hline$\hat{C}_{8}$ & Doses I & $v s$ & Doses II & SI e DUI & $v s$ & SII e DUII \\
\hline$\hat{C}_{9}$ & Testemunha & $v s$ & Seqüenciais & Testemunha sem aplicação & $v s$ & SI e SII \\
\hline$\hat{C}_{10}$ & Testemunha & vs & Doses Únicas & Testemunha sem aplicação & vs & DUI e DUII \\
\hline
\end{tabular}

Tabela 5 - Produção relativa de matéria seca de nódulos acumulados (MSNT) em 20 variedades de soja RR, em função de cinco modalidades de aplicação com glyphosate

\begin{tabular}{|c|c|c|c|c|c|c|c|c|c|c|}
\hline \multicolumn{11}{|c|}{ Matéria seca de nódulo total acumulado $(\%)^{1 / /}$} \\
\hline Variedade & $\begin{array}{c}\text { BRS } 245 \\
\text { RR }\end{array}$ & $\begin{array}{c}\text { MSOY } \\
8151 \text { RR }\end{array}$ & $\begin{array}{c}\text { AL } 83 \\
\text { Tropical } \\
\text { RR }\end{array}$ & $\begin{array}{l}\text { CD } 214 \\
\text { RR }\end{array}$ & $\begin{array}{c}\text { BRS } 242 \\
\text { RR }\end{array}$ & $\begin{array}{c}\text { BRS } 244 \\
\text { RR }\end{array}$ & $\begin{array}{l}\text { CD } 219 \\
\text { RR }\end{array}$ & $\begin{array}{c}\text { MSOY } \\
8008 \text { RR }\end{array}$ & $\begin{array}{c}\text { Anta } \\
\text { RR }\end{array}$ & $\begin{array}{c}\text { BRS } 243 \\
\text { RR }\end{array}$ \\
\hline Test $^{4 /}$ & $100,0 \mathrm{aA}^{\frac{3}{}}$ & $100,0 \mathrm{aA}$ & $100,0 \mathrm{aA}$ & $100,0 \mathrm{aA}$ & $100,0 \mathrm{aA}$ & $100,0 \mathrm{aA}$ & $100,0 \mathrm{aA}$ & $100,0 \mathrm{aA}$ & $100,0 \mathrm{aA}$ & $100,0 \mathrm{aA}$ \\
\hline $\mathrm{SI}^{\frac{5}{}}$ & $83,7 \mathrm{aA}$ & $89,6 \mathrm{aA}$ & $72,1 \mathrm{aA}$ & $85,1 \mathrm{aA}$ & $78,8 \mathrm{aA}$ & $79,1 \mathrm{aA}$ & $82,7 \mathrm{bA}$ & $73,1 \mathrm{bA}$ & $78,2 \mathrm{bA}$ & $82,0 \mathrm{aA}$ \\
\hline $\mathrm{DUI}^{6 /}$ & $78,1 \mathrm{aA}$ & $80,9 \mathrm{aA}$ & $90,3 \mathrm{aA}$ & $92,4 \mathrm{aA}$ & $66,9 \mathrm{aA}$ & $73,5 \mathrm{aA}$ & $70,6 \mathrm{bA}$ & $81,5 \mathrm{bA}$ & $62,3 \mathrm{bA}$ & $84,5 \mathrm{aA}$ \\
\hline $\mathrm{SIII}^{7 /}$ & $89,2 \mathrm{aA}$ & $83,2 \mathrm{aA}$ & $80,1 \mathrm{aA}$ & $77,0 \mathrm{aA}$ & $78,7 \mathrm{aA}$ & $81,2 \mathrm{aA}$ & $70,3 \mathrm{bA}$ & $71,5 \mathrm{bA}$ & $69,2 \mathrm{bA}$ & $86,8 \mathrm{aA}$ \\
\hline $\mathrm{DUII}^{8 /}$ & $76,6 \mathrm{aA}$ & $84,5 \mathrm{aA}$ & $76,0 \mathrm{aA}$ & $83,0 \mathrm{aA}$ & $83,0 \mathrm{aA}$ & $81,7 \mathrm{aA}$ & $71,3 \mathrm{bA}$ & $61,6 \mathrm{bB}$ & $67,5 \mathrm{bA}$ & $86,3 \mathrm{aA}$ \\
\hline $\mathrm{MA}^{\frac{3}{3}}$ Variedade & $\begin{array}{c}\text { MSOY } \\
6001 \text { RR }\end{array}$ & $\begin{array}{c}\text { Cristalina } \\
\text { RR }\end{array}$ & $\begin{array}{c}\text { BRS } 247 \\
\text { RR }\end{array}$ & $\begin{array}{c}\text { MSOY } \\
9000 \mathrm{RR}\end{array}$ & $\begin{array}{l}\text { CD } 213 \\
\text { RR }\end{array}$ & $\begin{array}{l}\text { MSOY } \\
8100 \text { RR }\end{array}$ & $\begin{array}{l}\text { Vali osa } \\
\text { RR }\end{array}$ & $\begin{array}{c}\text { MSOY } \\
8000 \text { RR }\end{array}$ & $\begin{array}{c}\text { BRS } 246 \\
\text { RR }\end{array}$ & NI \\
\hline Test $^{4 /}$ & $100,0 \mathrm{aA}$ & $100,0 \mathrm{aA}$ & $100,0 \mathrm{aA}$ & $100,0 \mathrm{aA}$ & $100,0 \mathrm{aA}$ & $100,0 \mathrm{aA}$ & $100,0 \mathrm{aA}$ & $100,0 \mathrm{aA}$ & $100,0 \mathrm{aA}$ & $100,0 \mathrm{aA}$ \\
\hline $\mathrm{SI}^{\frac{5 /}{1}}$ & $74,9 \mathrm{bA}$ & $70,7 \mathrm{bA}$ & $77,3 \mathrm{aA}$ & $85,2 \mathrm{aA}$ & $72,2 \mathrm{bA}$ & $66,0 \mathrm{bA}$ & $71,4 \mathrm{bA}$ & $70,4 \mathrm{bA}$ & $74,3 \mathrm{aA}$ & $71,9 \mathrm{bA}$ \\
\hline $\mathrm{DUI}^{-\prime}$ & $69,3 \mathrm{bA}$ & $86,6 \mathrm{aA}$ & $81,3 \mathrm{aA}$ & $91,0 \mathrm{aA}$ & $64,2 \mathrm{bA}$ & $71,1 \mathrm{bA}$ & $70,9 \mathrm{bA}$ & $72,5 \mathrm{bA}$ & $86,6 \mathrm{aA}$ & $73,6 \mathrm{bA}$ \\
\hline $\mathrm{SII}^{7 /}$ & $68,8 \mathrm{bA}$ & $70,2 \mathrm{bA}$ & $73,2 \mathrm{aA}$ & $85,6 \mathrm{aA}$ & $49,0 \mathrm{cA}$ & $66,1 \mathrm{bA}$ & $67,7 \mathrm{bA}$ & $68,2 \mathrm{bA}$ & $88,5 \mathrm{aA}$ & $71,7 \mathrm{bA}$ \\
\hline DUII $^{8 /}$ & $57,0 \mathrm{bB}$ & $74,2 \mathrm{bA}$ & $82,8 \mathrm{aA}$ & $78,8 \mathrm{aA}$ & $38,6 \mathrm{cB}$ & $72,5 \mathrm{bA}$ & $73,2 \mathrm{bA}$ & $69,0 \mathrm{bA}$ & $89,9 \mathrm{aA}$ & $69,3 \mathrm{bA}$ \\
\hline
\end{tabular}

1/ Porcentagem em relação à testemunha ${ }^{2 /}$ Médias seguidas de letras iguais, minúsculas nas colunas e maiúsculas nas linhas, não diferem estatisticamente pelo teste de Scott-Knott a $5 \%$ de significância ${ }^{3 /} \mathrm{MA}$ : modalidade de aplicação; ${ }^{4 /}$ Test $=$ testemunha sem herbicida; ${ }^{5} / \mathrm{SI}=$ aplicação seqüencial I; ${ }^{6 /} \mathrm{DUI}=$ dose única I; ${ }^{7 /} \mathrm{SII}=$ aplicação seqüencial II; $\stackrel{8}{8} \mathrm{DUII}=$ dose única II. 
Bohrer \& Hungria (1998) correlacionaram MSNT com a quantidade de nitrogênio total da parte aérea e encontraram correlações elevadas e significativas. Uma vez que o nitrogênio total obtido pela parte aérea das plantas é determinante na produtividade de grãos e no teor de proteínas destes e que este nitrogênio provém basicamente da atividade dos nódulos, é possivel inferir que os resultados aqui apresentados possam contribuir na seleção de materiais menos sensiveis à aplicação de glyphosate.

O número de nódulos acumulados por vaso (NN) (Tabela 6) foi reduzido pelas quatro modalidades de aplicação em intensidade semelhante em relação às respectivas testemunhas para os cultivares AL 83 TROPICAL RR, CD 214 RR, BRS 244 RR, CD 219 RR, MSOY 8008 RR, ANTA RR, MSOY 6001 RR, CRISTALINA RR, BRS 247 RR, MSOY 8100 RR, VALIOSA RR, MSOY 8000 RR e NI. O cultivar CD 213 RR foi o mais afetado pelas aplicações nessa variável, apresentando reduções de até $68,1 \%$ para as modalidades de aplicação com dose maior (SII e DUII), 39,8\% para DUI e $28,3 \%$ para SI. BRS 242 RR apresentou redução em todas as modalidades de aplicação, com exceção da DUII. Uma hipótese para essa resposta diferencial na dose única maior pode ser a diferença de quatro dias em relação à aplicação da DUI.

A comparação entre os cultivares apresentou diferença nas aplicações com dose única (DUI e DUII) e na aplicação seqüencial com dose maior (SII). Na aplicação com dose única menor (DUI), os cultivares BRS 244 RR, CD 219 RR, MSOY 8008 RR, ANTA RR, MSOY 6001 RR, CD 213 RR, MSOY 8100 RR, VALIOSA RR, MSOY 8000 RR e NI apresentaram maior redução do número de nódulos em relação aos outros. Na aplicação seqüencial com dose maior (SII), os cultivares CD 214 RR, CD 219 RR, MSOY 8008 RR, ANTA RR, MSOY 6001 RR, CRISTALINA RR, BRS 247 RR, CD 213 RR, MSOY 8100 RR, VALIOSA RR, MSOY 8000 RR e NI tiveram maior redução em relação aos outros para esta variável. Para aplicação com dose única maior (DUII), formaram-se três grupos. Os cultivares AL 83 TROPICAL RR, CD 219 RR, MSOY 8008 RR, ANTA RR, MSOY 6001 RR, MSOY 9000 RR, MSOY 8100 RR e MSOY 8000 $\mathrm{RR}$ formaram o grupo intermediário de redução para esta variável e apresentaram menor diminuição de número de nódulos em relação aos cultivares CD 213 RR e NI e maior redução dessa variável em relação aos demais.

Tabela 6 - Produção relativa de número de nódulos (NN) em 20 variedades de soja RR, em função de cinco modalidades de aplicação com glyphosate

\begin{tabular}{|c|c|c|c|c|c|c|c|c|c|c|}
\hline \multicolumn{11}{|c|}{ Número de nódulo acumulado (\%) ${ }^{1 /}$} \\
\hline $\mathrm{MA}^{\frac{3}{}}$ & $\begin{array}{c}\text { BRS } 245 \\
\text { RR }\end{array}$ & $\begin{array}{c}\text { MSOY } \\
8151 \text { RR }\end{array}$ & $\begin{array}{c}\text { AL } 83 \\
\text { Tropical } \\
\text { RR }\end{array}$ & $\begin{array}{c}\text { CD } 214 \\
\text { RR }\end{array}$ & $\begin{array}{c}\text { BRS } 242 \\
\text { RR }\end{array}$ & $\begin{array}{l}\text { BRS } 244 \\
\text { RR }\end{array}$ & $\begin{array}{c}\text { CD } 219 \\
\text { RR }\end{array}$ & $\begin{array}{c}\text { MSOY } \\
8008 \text { RR }\end{array}$ & $\begin{array}{c}\text { ANTA } \\
\text { RR }\end{array}$ & $\begin{array}{c}\text { BRS } 243 \\
\text { RR }\end{array}$ \\
\hline Test $^{4 /}$ & $100,0 \mathrm{aA}^{\underline{2} /}$ & $100,0 \mathrm{aA}$ & $100,0 \mathrm{aA}$ & $100,0 \mathrm{aA}$ & $100,0 \mathrm{aA}$ & $100,0 \mathrm{aA}$ & $100,0 \mathrm{aA}$ & $100,0 \mathrm{aA}$ & $100,0 \mathrm{aA}$ & $100,0 \mathrm{aA}$ \\
\hline $\mathrm{SI}^{\mathrm{5} /}$ & $82,6 \mathrm{aA}$ & $80,8 \mathrm{aA}$ & $67,1 \mathrm{bA}$ & $74,3 \mathrm{bA}$ & $79,5 \mathrm{bA}$ & $72,3 \mathrm{bA}$ & $74,5 \mathrm{bA}$ & $66,5 \mathrm{bA}$ & $68,1 \mathrm{bA}$ & $75,6 \mathrm{aA}$ \\
\hline $\mathrm{DUI}^{6 /}$ & $79,3 \mathrm{aA}$ & $79,7 \mathrm{aA}$ & $80,2 \mathrm{bA}$ & $82,3 \mathrm{bA}$ & $74,0 \mathrm{bA}$ & $72,3 \mathrm{bB}$ & $70,2 \mathrm{bB}$ & $71,7 \mathrm{bB}$ & $60,8 \mathrm{bB}$ & $83,6 \mathrm{aA}$ \\
\hline $\mathrm{SII}^{7 /}$ & $87,1 \mathrm{aA}$ & $78,7 \mathrm{aA}$ & $73,0 \mathrm{bA}$ & $68,1 \mathrm{bB}$ & $75,1 \mathrm{bA}$ & $79,2 \mathrm{bA}$ & $62,4 \mathrm{bB}$ & $63,0 \mathrm{bB}$ & $62,5 \mathrm{bB}$ & $90,9 \mathrm{aA}$ \\
\hline DUII $^{8 /}$ & $78,6 \mathrm{aA}$ & $84,3 \mathrm{aA}$ & $62,2 \mathrm{bB}$ & $73,9 \mathrm{bA}$ & $97,0 \mathrm{aA}$ & $79,6 \mathrm{bA}$ & $63,6 \mathrm{bB}$ & $52,9 \mathrm{bB}$ & $65,3 \mathrm{bB}$ & $80,4 \mathrm{aA}$ \\
\hline $\mathrm{MA}^{3 /}$ & $\begin{array}{c}\text { MSOY } \\
6001 \text { RR }\end{array}$ & $\begin{array}{c}\text { Cristalina } \\
\text { RR }\end{array}$ & $\begin{array}{c}\text { BRS } 247 \\
\text { RR }\end{array}$ & $\begin{array}{c}\text { MSOY } \\
9000 \mathrm{RR}\end{array}$ & $\begin{array}{c}\text { CD } 213 \\
\text { RR }\end{array}$ & $\begin{array}{c}\text { MSOY } \\
8100 \text { RR }\end{array}$ & $\begin{array}{c}\text { Valiosa } \\
\text { RR }\end{array}$ & $\begin{array}{c}\text { MSOY } \\
8000 \text { RR }\end{array}$ & $\begin{array}{c}\text { BRS } 246 \\
\text { RR }\end{array}$ & NI \\
\hline Test $^{4 /}$ & $100,0 \mathrm{aA}$ & $100,0 \mathrm{aA}$ & $100,0 \mathrm{aA}$ & $100,0 \mathrm{aA}$ & $100,0 \mathrm{aA}$ & $100,0 \mathrm{aA}$ & $100,0 \mathrm{aA}$ & $100,0 \mathrm{aA}$ & $100,0 \mathrm{aA}$ & $100,0 \mathrm{aA}$ \\
\hline $\mathrm{SI}^{\mathrm{5} /}$ & $76,1 \mathrm{bA}$ & $67,9 \mathrm{bA}$ & $67,1 \mathrm{bA}$ & $81,0 \mathrm{aA}$ & $71,7 \mathrm{bA}$ & $56,5 \mathrm{bA}$ & $69,1 \mathrm{bA}$ & $59,9 \mathrm{bA}$ & $68,6 \mathrm{aA}$ & $65,2 \mathrm{bA}$ \\
\hline $\mathrm{DUI}^{6 /}$ & $63,2 \mathrm{bB}$ & $79,8 \mathrm{bA}$ & $77,9 \mathrm{bA}$ & $82,4 \mathrm{aA}$ & $60,2 \mathrm{cB}$ & $64,4 \mathrm{bB}$ & $60,9 \mathrm{bB}$ & $60,2 \mathrm{bB}$ & $92,2 \mathrm{aA}$ & $63,6 \mathrm{bB}$ \\
\hline $\mathrm{SII}^{7 /}$ & $65,3 \mathrm{bB}$ & $71,0 \mathrm{bB}$ & $68,9 \mathrm{bB}$ & $82,4 \mathrm{aA}$ & $42,1 \mathrm{~dB}$ & $57,1 \mathrm{bB}$ & $68,4 \mathrm{bB}$ & $61,5 \mathrm{bB}$ & $84,6 \mathrm{aA}$ & $62,2 \mathrm{bB}$ \\
\hline $\mathrm{DUII}^{\frac{8 /}{}}$ & $60,2 \mathrm{bB}$ & $73,2 \mathrm{bA}$ & $75,2 \mathrm{bA}$ & $67,5 \mathrm{aB}$ & $31,9 \mathrm{dC}$ & $63,5 \mathrm{bB}$ & $70,7 \mathrm{bA}$ & $63,4 \mathrm{bB}$ & $84,5 \mathrm{aA}$ & $47,7 \mathrm{bC}$ \\
\hline
\end{tabular}

1/ Porcentagem em relação à testemunha; ²/Médias seguidas de letras iguais, minúsculas nas colunas e maiúsculas nas linhas, não diferem estatisticamente pelo teste de Scott-Knott a 5\%; ㄹ MA: modalidade de aplicação; ${ }^{4 /}$ Test $=$ testemunha sem herbicida; ${ }^{5 /} \mathrm{SI}=$ aplicação seqüencial I; ${ }^{6 /} \mathrm{DUI}=$ dose única I; ${ }^{7 /} \mathrm{SII}=$ aplicação seqüencial II; $\stackrel{8 /}{\mathrm{DUII}}=$ dose única II. 
Estudos realizados por Reddy et al. (2000) demonstraram a redução do número de nódulos em soja convencional tratada com doses subletais de glyphosate e soja RR tratada com doses desse herbicida variando de 1,12 a 2,24 kg e.a. ha ${ }^{-1}$. Contudo, esses efeitos variaram de acordo com a época e dose de aplicação, e eles não se repetiram em trabalho realizado por Reddy \& Zablotowicz (2003), os quais encontraram redução do acúmulo de matéria seca de nódulos sem que houvesse redução no número de nódulos acumulados em cultivares de soja RR tratados com aplicações de $0,84 \mathrm{~kg}$ e.a. ha ${ }^{-1}$ de vários sais de glyphosate; da mesma forma, esses resultados também foram observados neste trabalho.

A matéria seca do sistema radicular (MSSR) (Tabela 7) foi afetada por pelo menos uma das modalidades de aplicação de glyphosate nas variedades BRS 245 RR, MSOY 8008 RR, ANTA RR, MSOY 6001 RR, CRISTALINA RR, BRS 247 RR, CD 213 RR e MSOY 8100 RR. A variedade ANTA RR teve a MSSR reduzida pelas duas modalidades de aplicação em dose única (DUI e DUII) em até $28,2 \%$, em relação à testemunha. A variedade MSOY 8100 RR apresentou redução $(32,4 \%)$ de MSSR apenas na aplicação seqüencial com dose maior de glyphosate (SII). O cultivar CRISTALINA RR apresentou reduções de até $23,1 \%$ para ambas as aplicações seqüenciais (SI e SII). Os cultivares BRS 245 RR, MSOY 8008 RR, MSOY 6001 RR, BRS 247 RR e CD 213 RR tiveram a MSSR reduzida em intensidade semelhante por todas as modalidades de aplicação de glyphosate (SI, SII, DUI e DUII) quando comparadas com suas respectivas testemunhas. Os cultivares MSOY 8151 RR, AL 83 TROPICAL RR, CD 214 RR, BRS 242 RR, BRS 244 RR, CD 219 RR, BRS 243 RR, MSOY 9000 RR, MSOY 8000 RR, BRS 246, VALIOSA e NI não tiveram a MSSR afetada por nenhuma das modalidades de aplicação com glyphosate.

Fazendo a comparação entre os cultivares, observa-se que BRS 245 RR, BRS 247 RR e CD $213 \mathrm{RR}$ foram mais afetados que os demais nas aplicações SII e DUII ao cultivar MSOY 8100 foi mais afetado que os demais apenas na modalidade de aplicação seqüencial maior (SII). Os cultivares CD 219 RR, MSOY 8008 RR, ANTA RR, MSOY 6001 RR e VALIOSA RR tiveram a MSSR mais reduzida que as demais variedades apenas na modalidade de aplicação com dose única maior (DUII). Os cultivares MSOY 8151 RR, AL 83 TROPICAL RR, CD 214 RR, BRS 242 RR, BRS 244 RR, BRS 243 RR, MSOY 9000 RR, MSOY 8000 RR, BRS 246 RR e NI apresentaram produção de MSSR semelhante entre

Tabela 7 - Produção relativa de matéria seca do sistema radicular (MSSR) em 20 variedades de soja RR, em função de cinco modalidades de aplicação com glyphosate

\begin{tabular}{|c|c|c|c|c|c|c|c|c|c|c|}
\hline \multicolumn{11}{|c|}{ Matéria seca do sistema radicular $(\%)^{1 /}$} \\
\hline Variedade & $\begin{array}{c}\text { BRS } 245 \\
\text { RR }\end{array}$ & $\begin{array}{c}\text { MSOY } \\
8151 \text { RR }\end{array}$ & $\begin{array}{c}\text { AL } 83 \\
\text { Tropical } \\
\text { RR }\end{array}$ & $\begin{array}{c}\text { CD } 214 \\
\text { RR }\end{array}$ & $\begin{array}{c}\text { BRS } 242 \\
\text { RR }\end{array}$ & $\begin{array}{c}\text { BRS } 244 \\
\text { RR }\end{array}$ & $\begin{array}{c}\text { CD } 219 \\
\text { RR }\end{array}$ & $\begin{array}{c}\text { MSOY } \\
8008 \text { RR }\end{array}$ & $\begin{array}{c}\text { ANTA } \\
\text { RR }\end{array}$ & $\begin{array}{c}\text { BRS } 243 \\
\text { RR }\end{array}$ \\
\hline Test $^{4 /}$ & $100,0 \mathrm{aA}^{\underline{2} /}$ & $100,0 \mathrm{aA}$ & $100,0 \mathrm{aA}$ & $100,0 \mathrm{aA}$ & $100,0 \mathrm{aA}$ & $100,0 \mathrm{aA}$ & $100,0 \mathrm{aA}$ & $100,0 \mathrm{aA}$ & $100,0 \mathrm{aA}$ & $100,0 \mathrm{aA}$ \\
\hline $\mathrm{SI}^{5 /}$ & $77,9 \mathrm{bA}$ & $81,0 \mathrm{aA}$ & $81,9 \mathrm{aA}$ & $96,3 \mathrm{aA}$ & $78,5 \mathrm{aA}$ & $77,4 \mathrm{aA}$ & $79,2 \mathrm{aA}$ & $80,9 \mathrm{bA}$ & $88,1 \mathrm{aA}$ & $91,0 \mathrm{aA}$ \\
\hline $\mathrm{DUI}^{\mathrm{G} /}$ & $84,9 \mathrm{bA}$ & $88,9 \mathrm{aA}$ & $96,7 \mathrm{aA}$ & $95,4 \mathrm{aA}$ & $80,1 \mathrm{aA}$ & $88,7 \mathrm{aA}$ & $84,3 \mathrm{aA}$ & $87,0 \mathrm{bA}$ & $73,9 \mathrm{bA}$ & $91,1 \mathrm{aA}$ \\
\hline $\mathrm{SII}^{\mathrm{7} /}$ & $69,6 \mathrm{bB}$ & $87,7 \mathrm{aA}$ & $94,6 \mathrm{aA}$ & $87,7 \mathrm{aA}$ & $86,6 \mathrm{aA}$ & $83,6 \mathrm{aA}$ & $82,1 \mathrm{aA}$ & $77,0 \mathrm{bA}$ & $86,6 \mathrm{aA}$ & $87,9 \mathrm{aA}$ \\
\hline DUII $^{8 / 2}$ & $73,6 \mathrm{bB}$ & $90,4 \mathrm{aA}$ & $85,8 \mathrm{aA}$ & $93,9 \mathrm{aA}$ & $86,0 \mathrm{aA}$ & $88,3 \mathrm{aA}$ & $80,7 \mathrm{aB}$ & $76,7 \mathrm{bB}$ & $71,8 \mathrm{bB}$ & $92,4 \mathrm{aA}$ \\
\hline $\mathrm{MA}^{\frac{3}{2}}$ & $\begin{array}{l}\text { MSOY } \\
6001 \text { RR }\end{array}$ & $\begin{array}{c}\text { Cristalina } \\
\text { RR }\end{array}$ & $\begin{array}{c}\text { BRS } 247 \\
\text { RR }\end{array}$ & $\begin{array}{c}\text { MSOY } \\
9000 \mathrm{RR}\end{array}$ & $\begin{array}{c}\text { CD } 213 \\
\text { RR }\end{array}$ & $\begin{array}{l}\text { MSOY } \\
8100 \text { RR }\end{array}$ & $\begin{array}{c}\text { Valiosa } \\
\text { RR }\end{array}$ & $\begin{array}{c}\text { MSOY } \\
8000 \text { RR }\end{array}$ & $\begin{array}{c}\text { BRS } 246 \\
\text { RR }\end{array}$ & NI \\
\hline Test $^{4 /}$ & $100,0 \mathrm{Aa}$ & $100,0 \mathrm{aA}$ & $100,0 \mathrm{aA}$ & $100,0 \mathrm{aA}$ & $100,0 \mathrm{aA}$ & $100,0 \mathrm{aA}$ & $100,0 \mathrm{aA}$ & $100,0 \mathrm{aA}$ & $100,0 \mathrm{aA}$ & $100,0 \mathrm{aA}$ \\
\hline $\mathrm{SI}^{\frac{5 /}{6}}$ & $73,7 \mathrm{Ba}$ & $85,1 \mathrm{bA}$ & $65,8 \mathrm{bA}$ & $89,6 \mathrm{aA}$ & $71,3 \mathrm{bA}$ & $86,4 \mathrm{aA}$ & $89,2 \mathrm{aA}$ & $84,2 \mathrm{aA}$ & $91,1 \mathrm{aA}$ & $81,9 \mathrm{aA}$ \\
\hline $\mathrm{DUI}^{6 /}$ & $79,1 \mathrm{Ba}$ & $98,2 \mathrm{aA}$ & $78,1 \mathrm{bA}$ & $90,0 \mathrm{aA}$ & $77,9 \mathrm{bA}$ & $83,0 \mathrm{aA}$ & $82,8 \mathrm{aA}$ & $81,7 \mathrm{aA}$ & $89,2 \mathrm{aA}$ & $93,3 \mathrm{aA}$ \\
\hline $\mathrm{SII}^{\mathrm{7} /}$ & $78,7 \mathrm{bA}$ & $76,9 \mathrm{bA}$ & $59,5 \mathrm{bB}$ & $89,0 \mathrm{aA}$ & $62,7 \mathrm{bB}$ & $67,6 \mathrm{bB}$ & $81,7 \mathrm{aA}$ & $79,2 \mathrm{aA}$ & $89,1 \mathrm{aA}$ & $85,6 \mathrm{aA}$ \\
\hline $\mathrm{DUII}^{8 /}$ & $65,7 \mathrm{bB}$ & $90,5 \mathrm{aA}$ & $71,0 \mathrm{bB}$ & $90,6 \mathrm{aA}$ & $74,2 \mathrm{bB}$ & $89,2 \mathrm{aA}$ & $77,7 \mathrm{aB}$ & $86,6 \mathrm{aA}$ & $95,0 \mathrm{aA}$ & $84,2 \mathrm{aA}$ \\
\hline
\end{tabular}

1/ Porcentagem em relação à testemunha; ${ }^{2}$ Médias seguidas de letras iguais, minúsculas nas colunas e maiúsculas nas linhas, não diferem

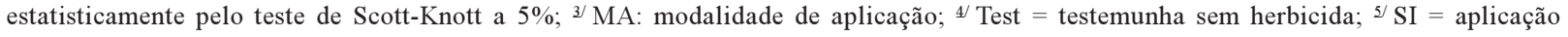
seqüencial I; ${ }^{6 /}$ DUI $=$ dose única I; ${ }^{7 /}$ SII = aplicação seqüencial II; $\underline{8} /$ DUII $=$ dose única II. 
si e superior em relação à dos demais cultivares.

Esses resultados concordam com os encontrados por King et al. (2001), quando estudaram o crescimento e a atividade da nitrogenase em diversos cultivares de soja RR e encontraram diferentes respostas dos cultivares para a variável MSSR, ora diminuindo o acúmulo de matéria seca, ora mantendo-o. Por outro lado, Reddy \& Zablotowicz (2003) encontraram resultados que diferem dos obtidos aqui, constatando recuperação da planta tratada por glyphosate 14 dias após a aplicação, equiparando sua MSSR com a da testemunha sem herbicida.

No tocante ao acúmulo de matéria seca da parte aérea (MSPA) (Tabela 8), pouco se observou de redução significativa das modalidades de aplicação em relação às respectivas testemunhas. De modo geral, as modalidades de aplicação de glyphosate tiveram menor efeito sobre a MSPA do que sobre a MSSR. Reduções significativas da MSPA ocorreram nos cultivares MSOY 8008 RR, CRISTALINA RR, BRS 247 RR e VALIOSA RR, os quais foram afetados pelas quatro modalidades de aplicação de glyphosate em intensidades semelhantes, em relação às suas respectivas testemunhas sem aplicação. Os cultivares CD 213 RR e AL 83 TROPICAL RR apresentaram redução nas modalidades de aplicação SI, SII e DUII para o primeiro cultivar e nas modalidades SI e DUII para o segundo, respectivamente. Comparações entre os cultivares não mostraram diferença significativa para nenhuma das modalidades de aplicação.

King et al. (2001) testaram o efeito do glyphosate sobre plantas totalmente dependentes da nodulação para obtenção de nitrogênio e plantas supridas com nitrogênio mineral. Observou-se decréscimo na biomassa da parte aérea das plantas supridas ou não com nitrogênio. Esse fato sugere um possivel efeito direto negativo do glyphosate sobre a produção de matéria seca da parte aérea, sem que a redução na nodulação seja a principal responsável por esse decréscimo. Isso demonstra que os resultados obtidos no presente trabalho, apesar de terem sido observados em apenas seis dos vinte cultivares testados, já foram constatados por outros autores. Reddy et al. (2000) também encontraram redução da matéria seca tanto da parte aérea quanto do sistema radicular de plantas de soja RR tratadas com glyphosate em comparação com a testemunha sem herbicida; no entanto, esses resultados só foram possíveis com o dobro da dose máxima utilizada

Tabela 8 - Produção relativa de matéria seca da parte aérea (MSPA) em 20 variedades de soja RR, em função de cinco modalidades de aplicação com glyphosate

\begin{tabular}{|c|c|c|c|c|c|c|c|c|c|c|}
\hline \multicolumn{11}{|c|}{ Matéria seca da parte aérea $(\%)^{1 /}$} \\
\hline $\mathrm{MA}^{\frac{3}{2}}$ Variedade & $\begin{array}{c}\text { BRS } 245 \\
\text { RR }\end{array}$ & $\begin{array}{c}\text { MSOY } \\
8151 \text { RR }\end{array}$ & $\begin{array}{l}\text { AL } 83 \\
\text { Tropical } \\
\text { RR }\end{array}$ & $\begin{array}{l}\text { CD } 214 \\
\text { RR }\end{array}$ & $\begin{array}{c}\text { BRS } 242 \\
\text { RR }\end{array}$ & $\begin{array}{c}\text { BRS } 244 \\
\text { RR }\end{array}$ & $\begin{array}{c}\text { CD } 219 \\
\text { RR }\end{array}$ & $\begin{array}{c}\text { MSOY } \\
8008 \text { RR }\end{array}$ & $\begin{array}{c}\text { ANTA } \\
\text { RR }\end{array}$ & $\begin{array}{c}\text { BRS } 243 \\
\text { RR }\end{array}$ \\
\hline Test $^{4 /}$ & $100,0 \mathrm{aA}^{2 /}$ & $100,0 \mathrm{aA}$ & $100,0 \mathrm{aA}$ & $100,0 \mathrm{aA}$ & $100,0 \mathrm{aA}$ & $100,0 \mathrm{aA}$ & $100,0 \mathrm{aA}$ & $100,0 \mathrm{aA}$ & $100,0 \mathrm{aA}$ & $100,0 \mathrm{aA}$ \\
\hline $\mathrm{SI}^{5 /}$ & $89,4 \mathrm{aA}$ & $87,7 \mathrm{aA}$ & $81,1 \mathrm{bA}$ & $92,0 \mathrm{aA}$ & $89,9 \mathrm{aA}$ & $80,6 \mathrm{aA}$ & $84,5 \mathrm{aA}$ & $82,9 \mathrm{bA}$ & $100,0 \mathrm{aA}$ & $94,4 \mathrm{aA}$ \\
\hline $\mathrm{DUI}^{\mathrm{G} /}$ & $88,1 \mathrm{aA}$ & $87,9 \mathrm{aA}$ & $99,3 \mathrm{aA}$ & $94,0 \mathrm{aA}$ & $85,7 \mathrm{aA}$ & $91,0 \mathrm{aA}$ & $83,4 \mathrm{aA}$ & $79,8 \mathrm{bA}$ & $94,7 \mathrm{aA}$ & $96,7 \mathrm{aA}$ \\
\hline $\mathrm{SIII}^{\mathrm{T} /}$ & $85,9 \mathrm{aA}$ & $88,1 \mathrm{aA}$ & $95,1 \mathrm{aA}$ & $87,2 \mathrm{aA}$ & $93,5 \mathrm{aA}$ & $90,8 \mathrm{aA}$ & $82,4 \mathrm{aA}$ & $84,5 \mathrm{bA}$ & $89,0 \mathrm{aA}$ & $90,9 \mathrm{aA}$ \\
\hline DUII $^{8 /}$ & $85,4 \mathrm{aA}$ & $89,1 \mathrm{aA}$ & $85,0 \mathrm{bA}$ & $88,1 \mathrm{aA}$ & $94,3 \mathrm{aA}$ & $86,1 \mathrm{aA}$ & $86,0 \mathrm{aA}$ & $74,5 \mathrm{bA}$ & $93,1 \mathrm{aA}$ & $92,4 \mathrm{aA}$ \\
\hline $\mathrm{MA}^{3 / 2}$ Variedade & $\begin{array}{c}\text { MSOY } \\
6001 \mathrm{RR}\end{array}$ & $\begin{array}{c}\text { Cristalina } \\
\text { RR }\end{array}$ & $\begin{array}{c}\text { BRS } 247 \\
\text { RR }\end{array}$ & $\begin{array}{c}\text { MSOY } \\
9000 \mathrm{RR}\end{array}$ & $\begin{array}{c}\text { CD } 213 \\
\text { RR }\end{array}$ & $\begin{array}{c}\text { MSOY } \\
8100 \text { RR }\end{array}$ & $\begin{array}{l}\text { VALI } \\
\text { OSA RR }\end{array}$ & $\begin{array}{l}\text { MSOY } \\
8000 \text { RR }\end{array}$ & $\begin{array}{c}\text { BRS } 246 \\
\text { RR }\end{array}$ & NI \\
\hline Test $^{4 /}$ & $100,0 \mathrm{aA}$ & $100,0 \mathrm{aA}$ & $100,0 \mathrm{aA}$ & $100,0 \mathrm{aA}$ & $100,0 \mathrm{aA}$ & $100,0 \mathrm{aA}$ & $100,0 \mathrm{aA}$ & $100,0 \mathrm{aA}$ & $100,0 \mathrm{aA}$ & $100,0 \mathrm{aA}$ \\
\hline $\mathrm{SI}^{5 /}$ & $77,7 \mathrm{aA}$ & $79,0 \mathrm{bA}$ & $78,9 \mathrm{bA}$ & $88,6 \mathrm{aA}$ & $88,1 \mathrm{bA}$ & $83,5 \mathrm{aA}$ & $83,2 \mathrm{bA}$ & $84,5 \mathrm{aA}$ & $88,6 \mathrm{aA}$ & $89,8 \mathrm{aA}$ \\
\hline $\mathrm{DUI}^{6 /}$ & $89,5 \mathrm{aA}$ & $88,8 \mathrm{bA}$ & $85,1 \mathrm{bA}$ & $90,2 \mathrm{aA}$ & $97,8 \mathrm{aA}$ & $90,5 \mathrm{aA}$ & $78,8 \mathrm{bA}$ & $87,3 \mathrm{aA}$ & $93,5 \mathrm{aA}$ & $95,0 \mathrm{aA}$ \\
\hline $\mathrm{SII}^{7 /}$ & $90,8 \mathrm{aA}$ & $85,5 \mathrm{bA}$ & $80,6 \mathrm{bA}$ & $87,6 \mathrm{aA}$ & $84,9 \mathrm{bA}$ & $84,9 \mathrm{aA}$ & $81,2 \mathrm{bA}$ & $85,7 \mathrm{aA}$ & $91,5 \mathrm{aA}$ & $89,2 \mathrm{aA}$ \\
\hline DUII $^{8 /}$ & $84,9 \mathrm{aA}$ & $79,7 \mathrm{bA}$ & $87,4 \mathrm{bA}$ & $86,2 \mathrm{aA}$ & $79,9 \mathrm{bA}$ & $90,3 \mathrm{aA}$ & $87,2 \mathrm{bA}$ & $81,9 \mathrm{aA}$ & $91,0 \mathrm{aA}$ & $84,6 \mathrm{aA}$ \\
\hline
\end{tabular}

1/Porcentagem em relação à testemunha; ̌ㅡ Médias seguidas de letras iguais, minúsculas nas colunas e maiúsculas nas linhas, não diferem estatisticamente pelo teste de Scott-Knott a 5\%; ${ }^{3 /} \mathrm{MA}$ : modalidade de aplicação; ${ }^{4 /}$ Test $=$ testemunha sem herbicida; ${ }^{5 /} \mathrm{SI}=$ aplicação seqüencial I; ${ }^{6}$ DUI $=$ dose única I; ${ }^{7 /} \mathrm{SII}=$ aplicação seqüencial II; $\stackrel{8}{2} \mathrm{DUII}=$ dose única II. 
neste trabalho, mostrando assim a existência de certa injúria que o glyphosate ocasionou sobre plantas de soja RR tratadas com doses maiores. Uma hipótese para as reduções ocorridas no acúmulo de MSPA e MSSR é o processo de degradação do glyphosate dentro da planta, que resulta na formação do ácido aminometilfosfônico (AMPA), conhecida fitotoxina (Duke et al., 2003; Reddy et al., 2004). Segundo Reddy et al. (2004), a formação de AMPA dentro da planta depende da taxa de glyphosate, do genótipo e das condições ambientais. Esses fatores que influenciam a formação de AMPA nas plantas explica por que alguns produtores confrontam com injúrias em soja geneticamente modificada e outros não, e por que os mesmos agricultores freqüentemente observam injúrias em um ano e em outros não.

CD 219 RR, ANTA RR, MSOY 6001 RR, BRS 247 RR, MSOY 8100 RR e VALIOSA RR apresentaram redução de três das quatro variáveis avaliadas em pelo menos uma das quatro modalidades de aplicação de glyphosate, quando comparados à testemunha. No entanto, não foi encontrado entre os cultivares um padrão de redução em relação às modalidades de aplicação, já que o cultivar ANTA RR teve a MSSR afetada apenas pelas aplicações em dose única (DUI e DUII). Por sua vez, MSOY 8100 apresentou redução da mesma variável apenas na modalidade de aplicação SII, demonstrando que cada cultivar responde de forma diferente às aplicações de glyphosate. Em MSOY 6001 RR, as quatro modalidades de aplicação causaram redução em relação à testemunha nas três variáveis citadas (MSNT, MSSR e NN), apresentando dessa forma mais um padrão de resposta em relação aos tratamentos.

Segundo os resultados obtidos, podem-se agrupar os cultivares em três grupos, segundo a tolerância às aplicações de glyphosate. Os cultivares BRS 242 RR, BRS 243 RR, BRS 244 RR, BRS 245 RR, BRS 246 RR, MSOY 8151 RR, MSOY 9000 RR e CD 214 RR apresentaram redução de nenhuma ou de uma das variáveis comparadas com suas respectivas testemunhas, sugerindo ser um grupo de maior tolerância às aplicações de glyphosate nas doses e modalidades de aplicação avaliadas neste trabalho. Os cultivares ANTA RR, CRISTALINA RR, MSOY 6001 RR, MSOY 8008 RR, MSOY 8100 RR, CD 213 RR, BRS 247 RR e VALIOSA RR foram os mais afetados pelas aplicações de glyphosate; pelo menos três das quatro variáveis analisadas (MSNT, MSSR, MSPA e NN) foram reduzidas por pelo menos uma das modalidades de aplicação, indicando se tratar de um grupo menos tolerante às aplicações de glyphosate. O grupo de tolerância intermediária conta com apenas quatro cultivares: AL 83 TROPICAL, CD 219 RR, MSOY 8000 RR e NI.

Os testes com contrastes permitiram classificar os efeitos das modalidades de aplicação e dos cultivares sobre as variáveis-resposta (MSSR, MSPA, MSNT e NN) estudadas (Tabela 9).

Os contrastes feitos entre modalidades de aplicação seqüenciais versus testemunha e doses únicas versus testemunha foram os únicos significativos para todas as variáveisresposta analisadas. Em ambos os casos houve superioridade das médias da testemunha em relação às modalidades de aplicação para as quatro variáveis estudadas (MSSR, MSPA, MSNT e NN). Isso sugere um efeito de redução das variáveis-resposta quando submetidas às modalidades de aplicação em relação à testemunha sem aplicação.

As comparações feitas entre os contrastes estabelecidos demonstraram diferença significativa para a MSSR apenas no contraste feito entre as aplicações seqüenciais e aplicações com dose única e os contrastes entre testemunha e modalidades de aplicação. Isso demonstra uma resposta melhor desta variável para as aplicações em dose única. Dessa forma, essa variável-resposta depende mais da modalidade de aplicação do que das doses de glyphosate, material genético ou grupo de maturação, o que possivelmente se deve à maior capacidade de metabolização do glyphosate e seus subprodutos em aplicação em dose única. Segundo Monquero (2003), diversos trabalhos demonstram as vantagens de aplicação seqüencial de glyphosate em relação a uma única aplicação sobre o controle de plantas daninhas de dificil controle. Esse fato sugere que o grau de injúria nas plantas é maior nas aplicações seqüenciais em relação à aplicação única, o que pode ser aplicado também aos resultados obtidos para soja RR neste trabalho em relação à MSSR.

Para MSPA, os contrastes que apresentaram significância foram entre grupos de 
Tabela 9 - Contrastes entre cultivares, grupos de maturação e modalidades de aplicação utilizadas no experimento, estimativas obtidas e probabilidade de significância para o teste $\mathrm{F}$ das variáveis-resposta matéria seca do sistema radicular (MSSR), matéria seca da parte aérea (MSPA), matéria seca de nódulos acumulados (MSNT) e número de nódulos acumulados (NN)

\begin{tabular}{|c|c|c|c|c|c|c|c|c|c|c|}
\hline \multicolumn{3}{|c|}{ Contraste } & \multicolumn{2}{|c|}{ MSSR } & \multicolumn{2}{|c|}{ MSPA } & \multicolumn{2}{|c|}{ MSNT } & \multicolumn{2}{|c|}{$\mathrm{NN}$} \\
\hline Coeficientes & $v s$ & Coeficientes & $\begin{array}{c}\text { Estimativas } \\
\text { dos } \\
\text { contrastes }\end{array}$ & $\operatorname{Pr}>f$ & $\begin{array}{c}\text { Estimativas } \\
\text { dos } \\
\text { contrastes }\end{array}$ & $\operatorname{Pr}>f$ & $\begin{array}{c}\text { Estimativas } \\
\text { dos } \\
\text { contrastes }\end{array}$ & $\operatorname{Pr}>f$ & $\begin{array}{c}\text { Estimativas } \\
\text { dos } \\
\text { contrastes }\end{array}$ & $\operatorname{Pr}>f$ \\
\hline Cultivares BRS & $v s$ & $\begin{array}{c}\text { Cultivares } \\
\text { Monsoy }\end{array}$ & $-0,24$ & 0,87 & $+2,43$ & 0,08 & $+5,05^{*}$ & 0,01 & $+8,80^{*}$ & $<0,01$ \\
\hline Cultivares BRS & $v s$ & Cultivares CD & $+0,16$ & 0,93 & $+1,30$ & 0,44 & $+7,70 *$ & $<0,01$ & $+11,85 *$ & $<0,01$ \\
\hline $\begin{array}{l}\text { Cultivares } \\
\text { Monsoy }\end{array}$ & $v s$ & Cultivares CD & $+0,40$ & 0,83 & $+1,13$ & 0,50 & $-2,65$ & 0,29 & $-3,04$ & 0,22 \\
\hline $\begin{array}{l}\text { Cultivares } \\
\text { precoces }\end{array}$ & $v s$ & $\begin{array}{c}\text { Cultivares } \\
\text { não-precoces }\end{array}$ & $+0,99$ & 0,46 & $+3,45^{*}$ & $<0,01$ & $+1,15$ & 0,51 & $+4,49^{*}$ & 0,01 \\
\hline SI & $v s$ & SII & $+1,86$ & 0,33 & $-1,25$ & 0,45 & $+2,11$ & 0,40 & $+1,03$ & 0,67 \\
\hline DUI & $v s$ & DUII & $+3,04$ & 0,11 & $+3,39 *$ & 0,04 & $+3,56$ & 0,15 & $+4,16$ & 0,09 \\
\hline Seqüenciais & $v s$ & Doses únicas & $-3,13 *$ & 0,02 & $-1,20$ & 0,31 & $+0,25$ & 0,88 & $-0,16$ & 0,92 \\
\hline Doses I & $v s$ & Doses II & $+2,45$ & 0,07 & $+1,07$ & 0,37 & $+2,83$ & 0,11 & $+2,59$ & 0,13 \\
\hline Testemunha & $v s$ & Seqüenciais & $+18,40 *$ & $<0,01$ & $+13,16^{*}$ & $<0,01$ & $+24,12 *$ & $<0,01$ & $+29,30 *$ & $<0,01$ \\
\hline Testemunha & $v s$ & Doses únicas & $+15,26 *$ & $<0,01$ & $+11,95 *$ & $<0,01$ & $+24,38^{*}$ & $<0,01$ & $+29,13 *$ & $<0,01$ \\
\hline
\end{tabular}

* Estimativas significativas pelo teste $\mathrm{F}$ a $5 \%$.

maturação e entre as duas modalidades de doses únicas, além dos citados anteriormente, sugerindo que a MSPA é dependente do grupo de maturação dos cultivares de soja e, se tratadas com aplicações em doses únicas, responde melhor às doses menores. Uma possível explicação para esse fato poderia ser a grande dependência da soja em relação ao ambiente em que está inserida, uma vez que a planta depende de fatores como temperatura e fotoperíodo para que seu desenvolvimento e floração sejam satisfatórios (Embrapa, 2006).

A variável MSNT demonstrou forte dependência do material genético, já que nenhum outro grupo testado pelos contrastes, além dos contrastes entre testemunhas e modalidades de aplicação, apresentou diferença significativa, demonstrando superioridade de acúmulo de MSNT nos cultivares BRS testados, em comparação aos cultivares MONSOY e CD. A importância do genótipo da soja para o bom desempenho do processo da fixação biológica do $\mathrm{N}_{2}$ já havia sido ressaltada desde os primeiros ensaios conduzidos no Brasil (Döbereiner \& Arruda, 1967). De acordo com Bohrer \& Hungria (1998), na ausência de uma avaliação continua das características relacionadas à fixação do $\mathrm{N}_{2}$ nos programas de melhoramento, podem ocorrer perdas genéticas em relação à capacidade simbiótica.
O número de nódulos (NN) foi afetado por dois dos fatores testados, além das modalidades de aplicação, em relação à testemunha. Um dos fatores foi o mesmo que influencia a MSNT, ou seja, o material genético dos cultivares em questão. O segundo fator (grupo de maturação) apresentou os cultivares precoces como mais favoráveis ao acúmulo de NN. Uma possivel hipótese para essa superioridade do grupo precoce em relação ao não-precoce pode estar relacionada ao período de início de formação dos nódulos. Nas precoces esse período possivelmente é menor que nas não-precoces, não sendo, portanto, influenciado pelas aplicações mais tardias, como as segundas aplicações seqüenciais e as duas aplicações em dose única.

Essas informações são de grande importância, pois associam cada parte do desenvolvimento da planta (variáveis-resposta) a uma determinada fonte de variação. Dessa forma, é possível inferir qual fonte de variação (material genético, grupo de maturação ou modalidades de aplicação) interfere em determinada variável-resposta (MSSR, MSPA, MSNT e NN). Tal análise permite inferências que podem contribuir na seleção de materiais menos sensiveis à aplicação de glyphosate.

De modo geral, MSSR é independente do grupo de maturação, variando segundo a 
modalidade de aplicação de glyphosate. MSPA apresenta tendência de melhor desempenho para os cultivares precoces em relação aos não-precoces. No caso do uso de aplicação em dose única, as doses menores causaram menores reduções nesta variável. Em MSNT e NN, observa-se um efeito evidente do material genético; o grupo de cultivares BRS apresentou melhor desempenho de nodulação em relação aos demais, após aplicações de glyphosate. Além disso, os cultivares mais precoces tendem a nodular em maior número quando expostos às situações aqui apresentadas.

Todas as doses e modalidades de aplicação de glyphosate causaram redução em todas as variáveis avaliadas. Os cultivares BRS 242 RR, BRS 243 RR, BRS 244 RR, BRS 245 RR, BRS 246 RR, MSOY 8151 RR, MSOY 9000 RR e CD $214 \mathrm{RR}$ apresentaram redução de nenhuma ou de uma das variáveis, comparadas com suas respectivas testemunhas, sugerindo ser um grupo de maior tolerância às aplicações de glyphosate nas doses e modalidades de aplicação avaliadas neste trabalho. Os efeitos do glyphosate sobre a soja RR são dependentes de fatores como variedade, grupo de maturação, época de aplicação e dose.

\section{AGRADECIMENTOS}

Ao Professor Dr. Osvaldo Ferrarese Filho, pelas sugestões na redação deste trabalho.

\section{LITERATURA CITADA}

ALAGAVADI, A. R.; REDDY, T. K. R. Effect of trifluralin on Rhizobium and its nodulation on groundnut. Pesticides, v. 20, n. 1, p. 27-30, 1986.

BELLALOUI, N. et al. Simulated glyphosate drift influences nitrate assimilation and nitrogen fixation in non-glyphosateresistant soybean. J. Agric. Food Chem., v. 54, n. 9 , p. 3357-3364, 2006.

BOHRER, T. R. J.; HUNGRIA, M. Avaliação de cultivares de soja quanto à fixação biológica do nitrogênio. Pesq.

Agropec. Bras., v. 33, n. 6, p. 937-952, 1998.

DELANNAY, X. et al. Yield evaluation of a glyphosatetolerant soybean line after treatment with glyphosate. Crop Sci., v. 35, n. 5, p. 1461-1467, 1995.

DÖBEREINER, J.; ARRUDA, N. B. Inter-relações entre variedades e nutrição na nodulação e simbiose da soja. Pesq. Agropec. Bras., v. 2, n. 2, p. 475-487, 1967.

Planta Daninha, Viçosa-MG, v. 26, n. 4, p. 831-843, 2008
DUKE, S. O.; RIMANDO, A. M. et al. Isoflavone, glyphosate, and aminomethylphosphonic acid levels in seeds of glyphosate-treated, glyphosate-resistant soybean. J. Agric. Food Chem., v. 51, n. 1, p. 340-344, 2003.

EBERBACH, P. L.; DOUGLAS, L. A. Herbicide effects on the growth and nodulation potential of Rhizobium trifolii with Trifolium subterraneum L. Plant Soil, v. 119, n. 1, p. $15-23,1989$.

EMPRESA BRASILEIRA DE PESQUISA

AGROPECUÁRIA - EMBRAPA. Exigências climáticas. In: Tecnologias de produção de soja - Paraná - 2007.

Londrina: 2006. 217 p.

FERREIRA, D. F. Análises estatísticas por meio do SISVAR para Windows versão 4.0. In: REUNIÃO ANUAL DA REGIÃO BRASILEIRA DA SOCIEDADE INTERNACIONAL DE BIOMETRIA, 2000, São Carlos Anais... São Carlos: Universidade Federal de São Carlos, 2000. p. $255-258$.

FISCHER, R. S. et al. Comparative action of glyphosate as a trigger of energy drain in Eubacteria. J. Bacteriol., v. 168, n. 3, p. 1147-1154, 1986.

HERNANDEZ, A.; GARCIA-PLAZAOLA, J. I.; BACERRIL, J. M. Glyphosate effects on phenolic metabolism of nodulated soybean (Glycine max L. Merril). J. Agric. Food Chem., v. 47, p. 2920-2925, 1999

JAWORSKI, E. G. Mode of action of Nphosphonomethylglycine: inhibition of aromatic amino acid biosynthesis. J. Agric. Food Chem., v. 20, n. 6, p. 1195 1198, 1972.

KAPUSTA, G.; ROUWENHORST, D. L. Interaction of selected pesticides and Rhizobium japonicum in pure culture and under field conditions. Agron. J., v. 65, n. 1, p. 112-115, 1973.

KING, C. A.; PURCELL, L. C.; VORIES, E. D. Plant growth and nitrogenase activity of glyphosate-tolerant soybean in response to foliar glyphosate applications. Agron. J., v. 93, n. 1, p. 176-186, 2001.

KISHINEVSKY, B. et al. Effects of some commercial herbicides on rhizobia and their symbiosis with peanuts. Weed Res., v. 28, p. 291-296, 1988.

LIU, C. M. et al. Degradation of the herbicide glyphosate by members of the family Rhizobiaceae. Appl. Environ. Microbiol., v. 57, n. 6, p. 1799-1804, 1991.

MARTENSSON, A. M. Effects of agrochemicals and heavy metals on fast-growing rhizobia and their symbiosis with small-seeded legumes. Soil Biol. Biochem., v. 24, n. 5, p.435-445, 1992. 
MONQUERO, P. A. Dinâmica populacional e mecanismos de tolerância de espécies de plantas daninhas ao herbicida glyphosate. 2003. 99 f. Tese (Doutorado - Programa de Pós-Graduação em Agronomia) Escola Superior de Agricultura "Luiz de Queiroz", Piracicaba, 2003.

MOORMAN, T. B. et al. Production of hydroxybenzoic acids by Bradyrhizobium japonicum strains after treatment with glyphosate. J. Agric. Food Chem., v. 40, n. 1, p. 289293, 1992.

OLIVEIRA JR., R. S. Mecanismos de ação de herbicidas. In: OLIVEIRA JR., R. S.; CONSTANTIN, J. Plantas daninhas e seu manejo. Guaíba: Agropecuária, 2001. p. 207-260.

REDDY, K. N.; HOAGLAND, R. E.; ZABLOTOWICZ, R. M. Effect of glyphosate on growth, chlorophyll content and nodulation in glyphosate-resistant soybeans (Glycine max) varieties. J. New Seeds, v. 2, n. 1, p. 37-52, 2000.
REDDY, K. N.; RIMANDO, A. M.; DUKE, S. O. Aminomethylphosphonic acid, a metabolite of glyphosate, causes injury in glyphosate-treated, glyphosate-resistant soybean. J. Agric. Food Chem., v. 52, n. 16, p. 5139-5143, 2004.

REDDY, K. N.; ZABLOTOWICZ, R. M. Glyphosateresistant soybean response to various salts of glyphosate and glyphosate accumulation in soybean nodules. Weed Sci., v. 51, p. 496-502, 2003.

SANTOS, J. B. et al. Efeitos de diferentes formulações comerciais de glyphosate sobre estirpes de Bradyrhizobium. Planta Daninha, v. 22, n. 2, p. 293-299, 2004

ZABLOTOWICZ, R. M.; REDDY, K. N. Impact of glyphosate on the Bradyrhizobium japonicum symbiosis with glyphosate-resistant transgenic soybean: a minireview. J. Environ. Qual., v. 33, n. 3, p. 825-831, 2004. 